\title{
The interferon type I signature towards prediction of non-response to rituximab in rheumatoid arthritis patients
}

\author{
Hennie G Raterman ${ }^{1 \dagger}$, Saskia Vosslamber ${ }^{2 \dagger}$, Sander de Ridder ${ }^{2}$, Michael T Nurmohamed ${ }^{3}$, Willem F Lems ${ }^{1,3}$,
} Maarten Boers ${ }^{1,3,4}$, Mark van de Wiel ${ }^{4}$, Ben AC Dijkmans ${ }^{1,3}$, Cornelis $L$ Verweij ${ }^{1,2^{*}}$ and Alexandre E Voskuyl ${ }^{1}$

\begin{abstract}
Introduction: B cell depletion therapy is efficacious in rheumatoid arthritis (RA) patients failing on tumor necrosis factor (TNF) blocking agents. However, approximately $40 \%$ to $50 \%$ of rituximab (RTX) treated RA patients have a poor response. We investigated whether baseline gene expression levels can discriminate between clinical nonresponders and responders to RTX.

Methods: In 14 consecutive RA patients starting on RTX (test cohort), gene expression profiling on whole peripheral blood RNA was performed by Illumina ${ }^{\circledR}$ HumanHT beadchip microarrays. Supervised cluster analysis was used to identify genes expressed differentially at baseline between responders and non-responders based on both a difference in 28 joints disease activity score ( $\triangle \mathrm{DAS} 28<1.2)$ and European League against Rheumatism (EULAR) response criteria after six months RTX. Genes of interest were measured by quantitative real-time PCR and tested for their predictive value using receiver operating characteristics (ROC) curves in an independent validation cohort $(n=26)$.
\end{abstract}

Results: Genome-wide microarray analysis revealed a marked variation in the peripheral blood cells between RA patients before the start of RTX treatment. Here, we demonstrated that only a cluster consisting of interferon (IFN) type I network genes, represented by a set of IFN type I response genes (IRGS), that is, LY6E, HERC5, IFI44L, ISG15, MXA, MXB, EPSTI1 and RSAD2, was associated with $\triangle \mathrm{DAS} 28$ and EULAR response outcome $(P=0.0074$ and $P=$ 0.0599, respectively). Based on the eight IRGS an IFN-score was calculated that reached an area under the curve (AUC) of 0.82 to separate non-responders from responders in an independent validation cohort of 26 patients using Receiver Operator Characteristics (ROC) curves analysis according to $\triangle \mathrm{DAS} 28<1.2$ criteria. Advanced classifier analysis yielded a three IRG-set that reached an AUC of 87\%. Comparable findings applied to EULAR non-response criteria.

Conclusions: This study demonstrates clinical utility for the use of baseline IRG expression levels as a predictive biomarker for non-response to RTX in RA.

\section{Introduction}

Rheumatoid arthritis (RA) is a systemic autoimmune disease characterized by chronic inflammation of the joints that may cause permanent cartilage and bone destruction. Currently, no curative treatment is available, and patients are subjected to a prolonged course of treatment.

\footnotetext{
* Correspondence: c.verweij@vumc.nl

+ Contributed equally

'Department of Rheumatology, VU University medical center, de Boelelaan

1117, Amsterdam, 1081HV, the Netherlands

Full list of author information is available at the end of the article
}

RA is marked by the presence of rheumatoid factor (RF) and/or anti-citrullinated protein autoantibodies (ACPA), which may precede the appearance of clinical symptoms of arthritis by many years [1,2]. Surface expressing RF Bcells may bind immune complexes and thereby serve a role as efficient antigen presenting cells that could lead to a break in T-cell tolerance against autoantigens [3]. In addition, an arthritogenic role for ACPA in experimental models of arthritis has been demonstrated [4,5]. Besides producers of auto-antibodies, B cells may contribute to

\section{Biomed Central}


disease pathogenesis through their role in antigen presentation, lymphoneogenesis and cytokine release [6]. Therefore, it was suggested that B-cells are essential players of the disturbed immune system, which fuelled interest in B-cells as drug target.

Rituximab (RTX) is a chimeric-human monoclonal antibody directed against the B cell marker CD20 that effectively depletes CD20-positive B cells. RTX is efficacious and safe in RA patients who are failing on TNF blocking agents [7-9]. Despite the effective depletion of circulating B cells in nearly all treated patients, clinical experience revealed that approximately $40 \%$ to $50 \%$ of RA patients do not respond to RTX $[8,9]$. Considering the progression of damage and the high costs of treatment with biologicals, identification of non-responders before start of treatment is highly desirable.

Clinical parameters such as baseline disability, number of previously used TNF blocking agents, and reason for ineffectiveness of anti-TNF treatment were found to be associated with non-response to RTX $[10,11]$. Whereas fluorescence activated cell sorter (FACS) studies revealed no association between $B$ cell numbers at baseline and clinical outcome, highly sensitive FACS technology suggested that the failure for complete B cell depletion at six months was associated with a poor response [12]. Pooled data from ten European registries (CARRERA) demonstrated that seropositive patients achieved significantly greater reductions in 28 joint disease activity score (DAS28) at six months than seronegative patients [13]. Others reported associations between BAFF/BLyS levels, Fc $\gamma$ RIII and IL-6 genotype, and Epstein-Barr virus genome in bone marrow and clinical outcome $[10,14,15]$. In addition, preliminary studies suggested an association between the expression level of transcripts in peripheral blood cells and clinical outcome [16,17]. Overall these findings have potential to provide a framework to select clinically relevant predictors but require validation and subsequent prognostic evaluation of clinical utility to warrant further development.

In the present study we focus on further analysis of transcript biomarkers in predicting response to RTX in order to determine the value of the proclaimed transcript markers and extend the transcript profile associated with response outcome. Key questions that we want to answer are: 1) Can previously reported transcript markers that are associated with clinical response be confirmed?; 2) What is the complexity of the transcript profile that is associated with responsiveness?; 3) Can we demonstrate the clinical utility of the transcript markers in an independent study? and 4) Can we optimize the transcript biomarker set to increase its predictive capacity? Therefore, we started whole genome transcript profiling of whole peripheral blood cells from patients prior to RTX treatment to study mRNA levels of all the genes in the genome simultaneously. Receiver Operating Characteristics (ROC)-curve analysis in an independent validation study was applied to demonstrate clinical utility and selection of the most optimal transcript biomarker set.

\section{Materials and methods \\ Patient recruitment}

Forty consecutive RA patients (14 for the test cohort and 26 for the validation cohort), according to the 1988 revised American College of Rheumatology criteria for the diagnosis of RA, attending the outpatient clinics of the VU University medical center and Jan van Breemen Research Institute|READE scheduled for RTX were followed prospectively [18]. Inclusion criteria for this study are according to the guidelines of the Dutch Society for Rheumatology for treatment with RTX, that is, active disease status (defined as DAS28 > 3.2 at treatment initiation) despite previous treatment with TNF-blocking agents, unless contraindicated in the opinion of the treating physician and previous treatment with methotrexate (MTX) and one other disease-modifying antirheumatic drug (DMARD). Moreover, patients needed to have a wash out period of at least one month from the last TNF blocker and at least six months follow up. All patients provided written informed consent and both participating clinics received approval by the local medical ethics committee. Demographic data and clinical characteristics are presented in Table 1.

\section{Treatment and clinical evaluation}

Patients received RTX 1,000 mg intravenously on days one and 15 in combination with clemastine (2 mg intravenously), methylprednisolone (100 mg intravenously) and acetaminophen (1,000 mg orally), as premedication. Four weeks after the first infusion and from 12 weeks on every three months, the DAS28 was assessed for disease activity status [19]. After six months the presence of a clinical nonresponse was defined in two ways: as $\triangle \mathrm{DAS} 28<1.2$, and as EULAR nonresponse (additionally requires DAS28 to be > 5.1) [20]. At these visits, serum and whole blood samples for RNA analysis were collected using PAXgene tubes (PreAnalytix, GmbH, Hilden, Germany. The use of concomitant DMARDs, prednisolone or NSAIDs during the study duration was permitted. Retreatment with RTX or treatment with other biological agents was prohibited during the six-month follow up.

\section{Laboratory Measurements \\ RNA isolation}

For RNA isolation, $2.5 \mathrm{ml}$ blood was drawn in PAXgene tubes, incubated for two hours at room temperature and stored at $-20^{\circ} \mathrm{C}$. Tubes were thawed overnight at room temperature prior to RNA isolation. Total RNA was isolated using Bio robot MDX (Qiagen, Benelux b.v., 
Table 1 Baseline characteristics and response-status in RTX treated RA patients.

\begin{tabular}{|c|c|c|c|}
\hline & $\begin{array}{l}\text { Test cohort } \\
(n=14)\end{array}$ & $\begin{array}{l}\text { Validation cohort } \\
\quad(n=26)\end{array}$ & $\begin{array}{l}\text { Total group } \\
(n=40)\end{array}$ \\
\hline \multicolumn{4}{|l|}{ Demographics } \\
\hline Age, years & $55 \pm 10$ & $59 \pm 11$ & $58 \pm 11$ \\
\hline Female, \% & 86 & 85 & 85 \\
\hline \multicolumn{4}{|l|}{ Disease characteristics } \\
\hline RA duration, years & 11 (5 to 21$)$ & $6(2$ to 16$)$ & 8 (3 to 17$)$ \\
\hline DAS28-score & $5.7 \pm 1.0$ & $5.8 \pm 1.2$ & $5.8 \pm 1.2$ \\
\hline $\mathrm{ESR}, \mathrm{mm} / \mathrm{hr}$ & 14 (9 to 31$)$ & 29 (13 to 50$)$ & 23 (12 to 42$)$ \\
\hline$C R P, m g / L$ & 9 (3 to 19$)$ & 14 (6 to 27$)$ & 12 (4 to 25$)$ \\
\hline IgM RF positive, $\%$ & 71 & 63 & 68 \\
\hline ACPA positive, $\%$ & 79 & 70 & 73 \\
\hline Erosive diseases, \% & 93 & 59 & 72 \\
\hline \multicolumn{4}{|l|}{ Medication } \\
\hline Previous biologicals, $\mathrm{n}$ & $2(1-2)$ & $2(1-2)$ & $2(1-2)$ \\
\hline > 1 Biological in history, \% & 50 & 50 & 50 \\
\hline Previous DMARDS, $\mathrm{n}$ & $4(4-5)$ & $3(3-4)$ & $4(3-4)$ \\
\hline Current prednisolone use, \% & 93 & 63 & 70 \\
\hline Prednisolone dosage, mg/day & $9(7-13)$ & $5(0-10)$ & $8(0-10)$ \\
\hline Current DMARD use, $\%$ & 93 & 77 & 83 \\
\hline Current statin use, \% & 21 & 13 & 18 \\
\hline \multicolumn{4}{|l|}{ Response } \\
\hline$\triangle \mathrm{DAS} 28$ & $-1.6(-2.7--0.5)$ & $-1.0(-1.5-0.2)$ & $-1.0(-2.0-0.3)$ \\
\hline $\begin{array}{l}\triangle D A S 28>1.2, \% \\
\text { EULAR responders, } \%\end{array}$ & $\begin{array}{l}57 \\
64\end{array}$ & $\begin{array}{l}65 \\
50\end{array}$ & $\begin{array}{l}63 \\
55\end{array}$ \\
\hline
\end{tabular}

Continuous variables are presented as mean with standard deviation or median with interquartile range where appropriate. ACPA, anti-cyclic citrullinated protein; CRP, C-reactive protein; DMARD, disease modifying anti-rheumatic drug; ESR, erythrocyte sedimentation rate; HCQ, hydroxychloroquine; MTX, methotrexate; RF, rheumatoid factor; SSZ, sulphasalazyne;

Venlo, The Netherlands) according to the manufacturer's instructions (PAXgene Blood RNA Mdx kit). Samples were cleaned from salts that may be present using the Qiagen RNA MinElute procedure according to the manufacturer's procedure (Qiagen, Venlo, The Netherlands). Total RNA concentration was measured using the Nanodrop spectrophotometer (Nanodrop Technologies, Wilmington, DE, USA) and RNA purity and integrity was verified using lab-on-chip technology (Agilent 2100 Bioanalyzer, Santa Clara, CA, USA).

\section{Microarray analysis}

Genome-wide transcriptome data were collected from peripheral blood cells of 14 patients prior to the start of RTX using baseline transcript data from 13 patients generated earlier for analysis of the pharmacological changes during RTX [21], supplemented with baseline data from an additional patient. Transcriptome data were generated using the HumanHT-12 v3 Expression BeadChip (Illumina, San Diego, CA. USA). In this process, 500 ng total RNA was used to synthesize biotin-labeled cRNA using the Illumina ${ }^{\circledR}$ TotalPrep ${ }^{\mathrm{TM}}$ RNA amplification kit (Ambion, Austin, TX, USA) and $750 \mathrm{ng}$ biotinylated cRNA was hybridized onto the Illumina beadchip (ServiceXS, Leiden, the Netherlands). Bead summary intensities were log2-transformed and normalized using quantile normalization [22,23]. All microarray data have been submitted to the Gene Expression Omnibus (GEO) under accession number GSE37107.

Hierarchical cluster analysis was used to categorize the data and TreeView was used to visualize differentially expressed genes [24]. Baseline characteristics of RA patients were expressed as mean (SD) or median (interquartile range (IQR)), where appropriate.

CDNA synthesis and quantitative real time PCR

Quantitative real-time PCR was performed on total RNA samples from the patients in the validation group $(n=26)$ using an ABI Prism 7900HT Sequence detection system (Applied Biosystems, Foster City, CA, USA). RNA (0.5 $\mu \mathrm{g})$ was reverse transcribed into cDNA using a RevertAid Hminus cDNA synthesis kit (MBI Fermentas, St. Leon-Rot, Germany) according to the manufacturer's instructions. Gene expression levels of one housekeeping gene, glyceraldehyde-3-phosphate dehydrogenase (GAPDH), and eight IRGs, that is, LY6E, HERC5, IFI44L, ISG15, MxA, MxB, EPSTI1 and RSAD2, were determined. To calculate arbitrary values of mRNA levels and to correct for differences in primer efficiencies for each gene a standard curve was constructed. Expression levels of target genes were expressed relative to GAPDH. 


\section{Calculation of interferon (IFN) score}

An IFN score was calculated as an average of expression levels of eight IRGs, namely, Ly6E, HERC5, IFI44L, ISG15, MxA, MxB, EPSTI1 and RSAD2, $\log 2$ based. Where indicated another set of IRGs was used. For univariate analysis and bivariate logistic regression analysis the IRG response data from both the test and validation cohort were median centered (as log2 transformed data) and combined to calculate the IFN score for further analysis.

\section{Statistical analysis}

Statistical analysis on microarray data was performed using Significance Analysis of Microarray (SAM), version 3.09 [25]. Two class paired analyses using SAM at a false discovery rate (FDR) of less than $5 \%$ between them were applied to search for single genes that were significantly differentially expressed between responders and nonresponders upon RTX treatment. Cluster analysis was used for the categorization of coordinately differentially expressed genes [24]. TreeView was used to visualize differentially expressed genes [26]. Ingenuity pathway analysis [27] was used for pathway level analysis.

Differences in gene expression levels of IFN type I network genes between patients with a $\triangle \mathrm{DAS} 28>1.2$ versus $\triangle \mathrm{DAS} 28<1.2$ or between EULAR good/moderate versus non-responders were analyzed using the Student unpaired $t$ test.

To select the best performing gene set of the eight IRGs for the ROC-curve performance we calculated the average expression of all combinations using $\mathrm{R}$ [28]. ROC curves were then constructed by applying the ROC package [29] available in Bioconductor. Responder status was based on the $\triangle \mathrm{DAS} 28$ non-responder status. The Area under the Curve (AUC) was calculated for each curve using the AUCi (integrated) function.

Stepwise regression analyses were performed to find associations between IRG gene expression levels and clinical response. Firstly, crude odds ratios (ORs) with 95\% confidence intervals $(\mathrm{CI})$ were calculated. Due to the number of patients included, it was not possible to perform multiple logistic regression analysis. Instead, the association between potential predictors and clinical outcome based on ORs with $95 \%$ CIs was compared to that for the IRG set. Subsequently, potential predictors for RTX response were added as covariates in a stepwise bivariate logistic analysis, that is, 1) seropositivity for IgM RF or ACPA, 2) lower number of previous TNF blocking agents, 3) use of lipid lowering drugs, 4) presence of erosions, 5) use of prednisolone, and 6) use of methotrexate.

All regression analyses were performed using SPSS version 15.0 (SPSS, Chicago, IL, USA).

$P$ values $<0.05$ are considered to be significant.

\section{Results}

Patient characteristics

A total of 40 patients were included, 14 of whom were subjected to genome-wide gene expression profiling (test cohort) and 26 of whom were used for validation (validation cohort). The clinical characteristics of these patients are shown in Table 1. Concomitant prednisolone use was lower in the validation cohort, although the daily dosage was not significantly different. Other characteristics were not significantly different between the test and validation cohort.

\section{Interferon Response Genes discriminate between non responders and responders}

In order to identify molecular markers that discriminate responders from non-responders to RTX in RA we analyzed the whole blood cell gene expression profile of 14 RA patients before the start of RTX treatment. Therefore, we searched for genes and/or gene signatures that were differentially expressed between responders (eight patients with a $\triangle \mathrm{DAS} 28>1.2$ after six months) and non-responders (six patients with a $\triangle \mathrm{DAS} 28 \leq 1.2$ ). Significant Analysis of Microarrays (SAM) at a FDR of less than $5 \%$ did not reveal differentially expressed genes at the single gene level. Subsequent analysis based on fold differences identified a total of 124 genes which differed at least two-fold in expression level (compared to the median expression level) in at least three out of the 14 patients. Supervised hierarchical cluster analysis, wherein patients were categorized as responders and non-responders based on $\triangle \mathrm{DAS} 28$, categorized these 124 genes into five groups, which represented different biological themes. Cluster one consists of genes from the IFN type I network which are related to IRF (IFN Regulatory Factor)-activation and IFN type I response activity. Genes of cluster two belong to processes such as DNA replication, recombination, and repair. Cluster three represents genes involved in protein synthesis, and cell-to-cell signaling and interaction. Genes involved in hematological system development and function and the immune cell trafficking network were characteristic for cluster four. Cluster five consists of genes mainly involved in immune regulation, such as NFAT regulation, complement system, B-cell development and the antigen presentation pathway

Next, we analyzed whether clusters of coordinately regulated genes are associated with clinical outcome (Figure 1A). The average expression levels of the genes per cluster were calculated and related to the clinical $\triangle$ DAS28 response status at six months following the start of RTX treatment. This analysis revealed a significant differential expression only of cluster one, consisting of IFN-type I response genes (IRGs). This cluster 


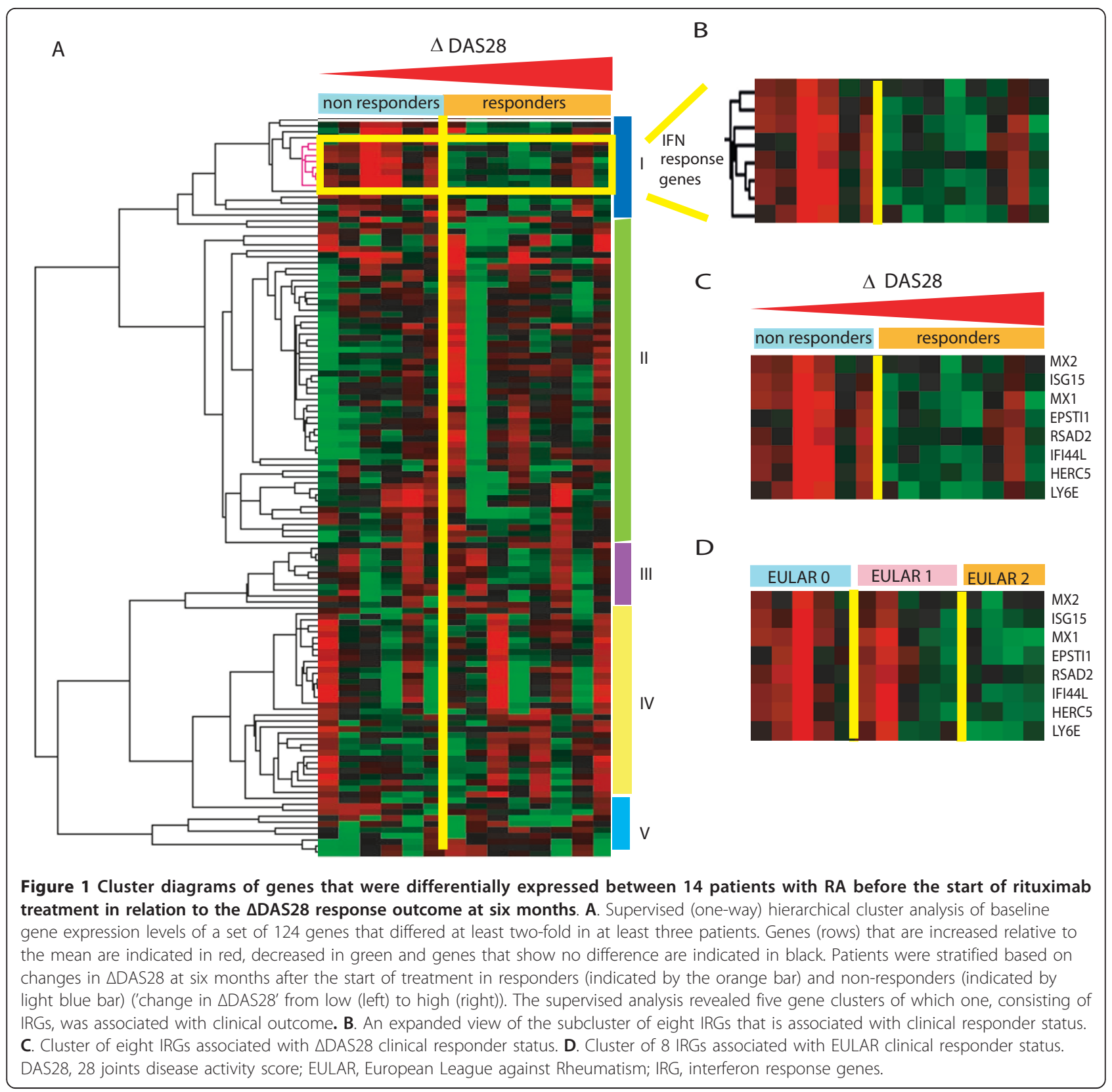

was represented by eight type I IRGs, namely, $L Y 6 E$, HERC5, IFI44L, ISG15, MxA, MxB, EPSTI1 and RSAD2, whose expression levels were highly correlated $(\mathrm{r}=0.91)$ (Figure 1B, C and 1D). A low expression of IRGs was associated with a good clinical response, whereas patients with increased expression of these genes exhibited a poor response $(P=0.0074)$ (Figure $1 C$ and $2 \mathrm{~A})$. Comparable results were observed when response status was assessed by the EULAR response criteria in good/ intermediate responders versus non-responders, although this association did not reach statistical significance $(P=0.0599)$ (Figure $1 \mathrm{D}$ and $2 \mathrm{~B}$ ).

\section{Prediction of response to RTX}

An important measure of the accuracy of the eight IRGset in separating the responders and non-responders is the ROC curve AUC analysis. Here we used the average expression values of the eight IRGs as an IFN score to construct an ROC curve using an independent cohort of 26 patients and calculated the AUC, which was evaluated as a predictor for non-response. At six months after the start of RTX treatment nine patients were classified as $\triangle \mathrm{DAS} 28$ responders and 17 as non-responders. This analysis revealed an AUC of 0.82 according to the six-month $\triangle \mathrm{DAS} 28$ criteria (Figure $3 \mathrm{~A}$ ). 


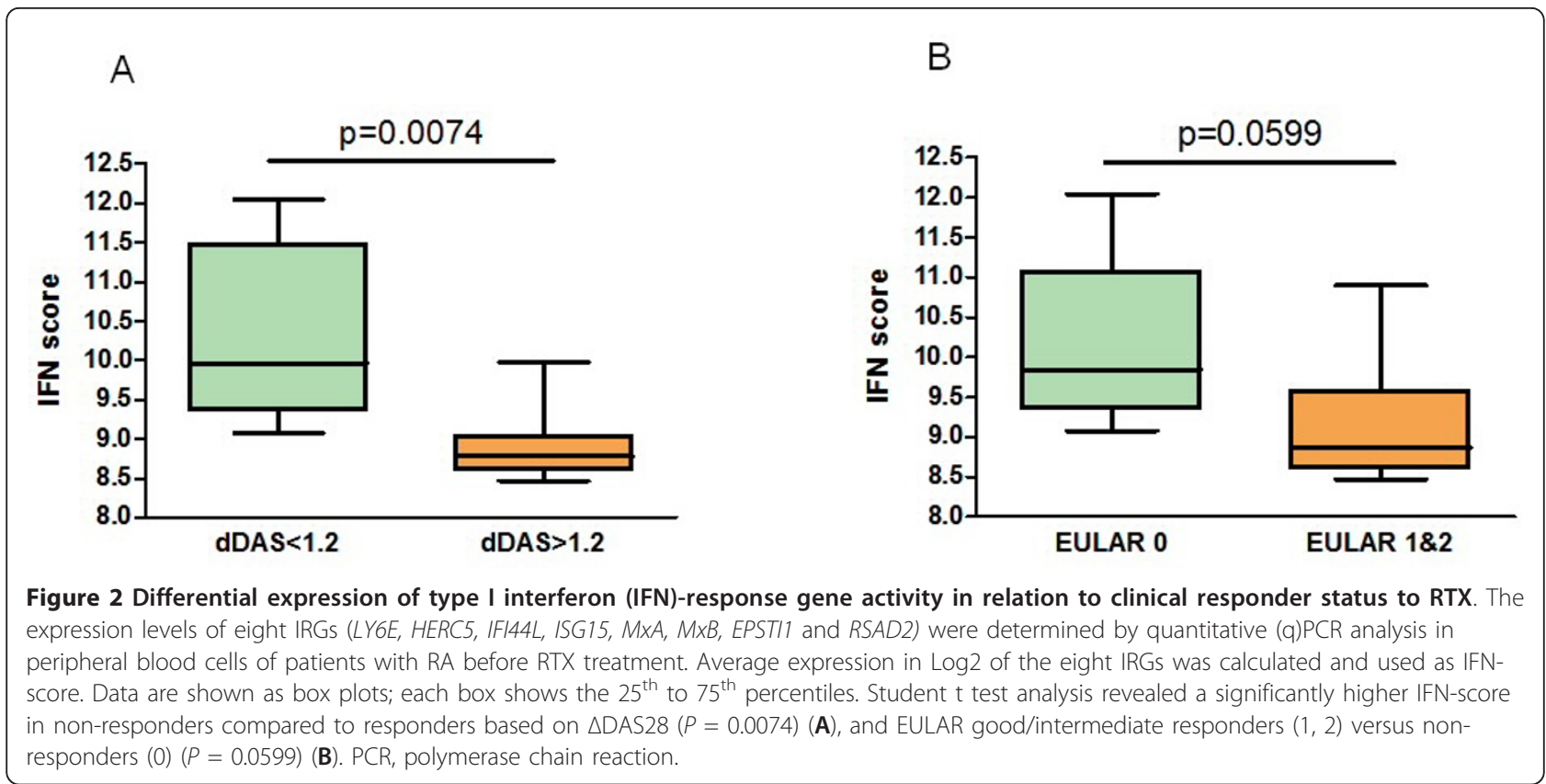

In order to select the most optimal gene set for response prediction based on ROC-curve AUC criteria we tested all combinations of the eight IRGs. For each combination, the average expression was calculated per patient and ROC curves were then constructed by applying the ROC package available in Bioconductor [29]. Responder status was based on the $\triangle \mathrm{DAS} 28$ non-responder status. Combinations of three, four or six specific IRGs (EPSTI1, RSAD2, $M x A$; or HERC5, RSAD2, MxA, LY6E; or HERC5, IFI44L, EPSTI1, RSAD2, MxA, LY6E, respectively) revealed the highest AUC of 0.87 (Figure 3B).

For both the eight IRG and three IRG sets we also calculated the ROC-curve AUC for the EULAR response outcome. These analyses revealed an AUC of 0.78 and 0.83 , respectively, demonstrating that these sets also have clinical utility for response prediction based on the EULAR response criteria (Figure $3 \mathrm{C}$ and $3 \mathrm{D}$ ).

Next, we studied the strength of the association between the eight IRG-set and non-response in the context of other potential predictors. We combined the data from the test and validation cohort. Univariate analysis showed that the eight gene sets were negatively associated with clinical response defined as $\triangle \mathrm{DAS} 28<1.2$ (OR: 0.25, 95\% C.I.: 0.09 to $0.70, P=0.008$, Nagelkerke $\left.R^{2} 0.379\right)$. Similar findings were made for the three gene set (OR: 0.25, 95\% C.I.: 0.10 to $0.65, P=0.004$, Nagelkerke $\left.R^{2} 0.42\right)$. The IRG sets outperform the association of other factors such as: 1 ) seropositivity for IgM RF or ACPA (OR: 1.6, 95\% C.I.: 0.92 to $2.83, P=0.097)$; 2) lower number of previous TNF blocking agents (OR: $0.29,95 \%$ C.I.: 0.077 to $1.06, P=$ 0.061 ); 3) use of lipid lowering drugs (OR:1.8, 95\% C.I.: 0.35 to $9.41, P=0.481$ ); 4 ) presence of erosions (OR:0.63,
95\% C.I.: 0.15 to $2.54, P=0.511) ; 5)$ use of prednisolone (OR:2.0, 95\% C.I.: 0.49 to $8.20, P=0.335$ ); and 6) use of MTX (OR: 0.3, 95\% C.I.: 0.076 to $1.15, P=0.078$ ). To determine if the IRG sets are predictors of clinical outcome independently from the other potential predictors, stepwise bivariate analysis was performed. These analyses revealed that adjustment for each single variate, that is, 1) seropositivity for IgM RF or ACPA; 2) more than one previous TNF blocking agent; 3 ) use of lipid lowering drugs; 4) presence of erosions; 5) prednisolone use; and 6) MTX use as well as age and gender had no significant influence on the association between IRGs (the eight or three gene set) and $\triangle \mathrm{DAS} 28$.

\section{Discussion}

B-cell depletion with the anti-CD20 antibody RTX has proven efficacious in RA $[7,8,30]$. However, clinical experience has revealed that approximately $40 \%$ to $50 \%$ of the patients show no clinical response. Genome-wide transcript profiling technology provides a very powerful and robust tool allowing an open-ended survey to identify comprehensively the fraction of genes that are differentially expressed between responders and non-responders before the start of treatment. This approach identified a selective group of genes that are all regulated by type I IFN that is significantly associated with clinical response. Our results not only confirm but also extend preliminary results reported previously [17]. In that study a candidategene approach was used to demonstrate an association between the expression of three randomly picked IRGs (Mx1, ISG15 and OAS1) by peripheral blood mononuclear cells (PBMC) and clinical outcome of RTX treatment. 


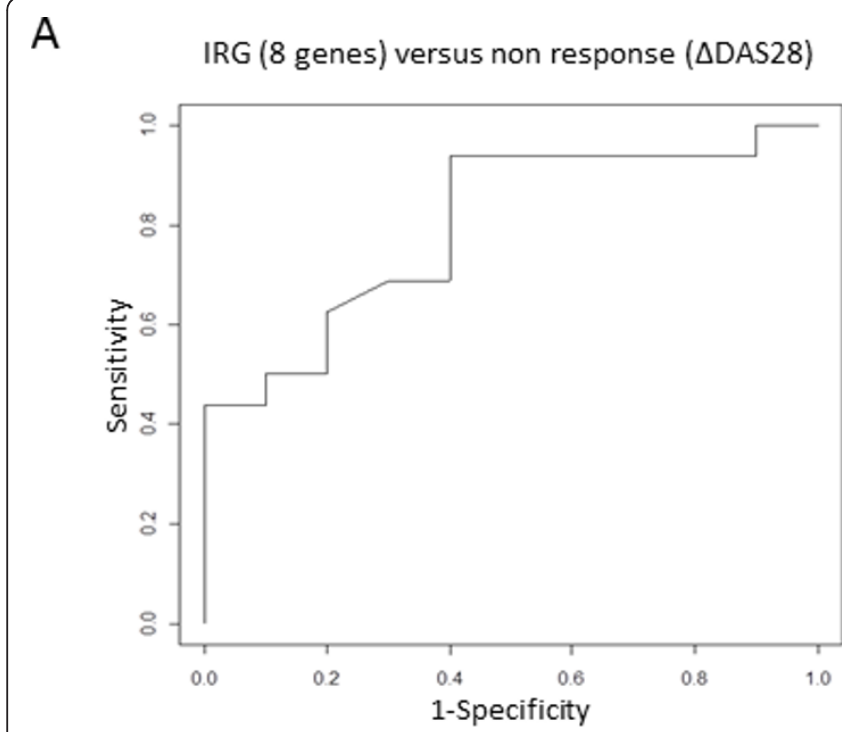

\section{B}

IRG (3 genes) versus non response ( $\triangle \mathrm{DAS} 28)$

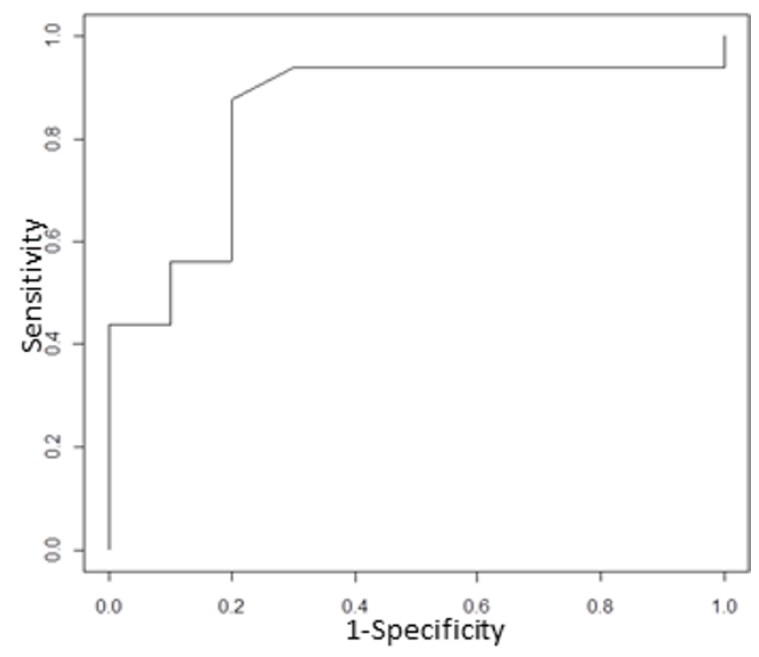

D
C

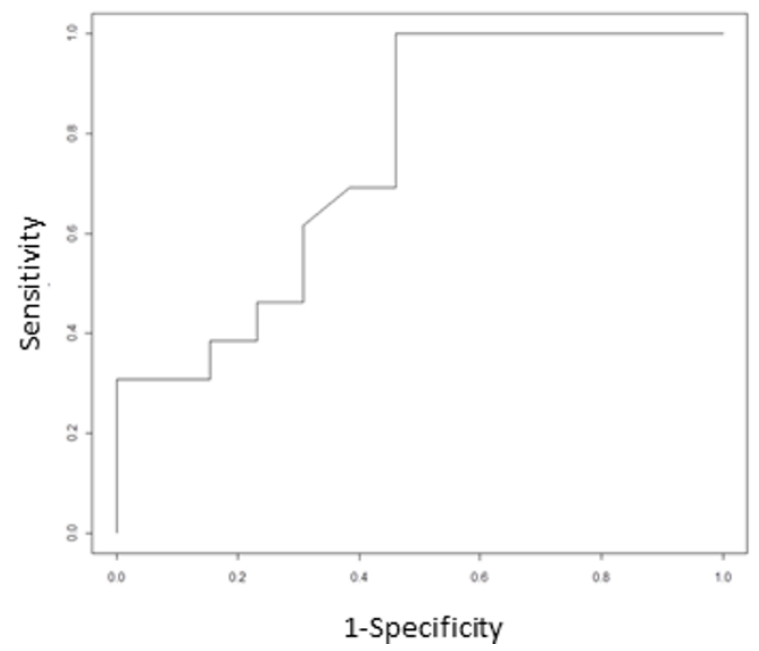

IRG (3 genes) versus non response (EULAR)

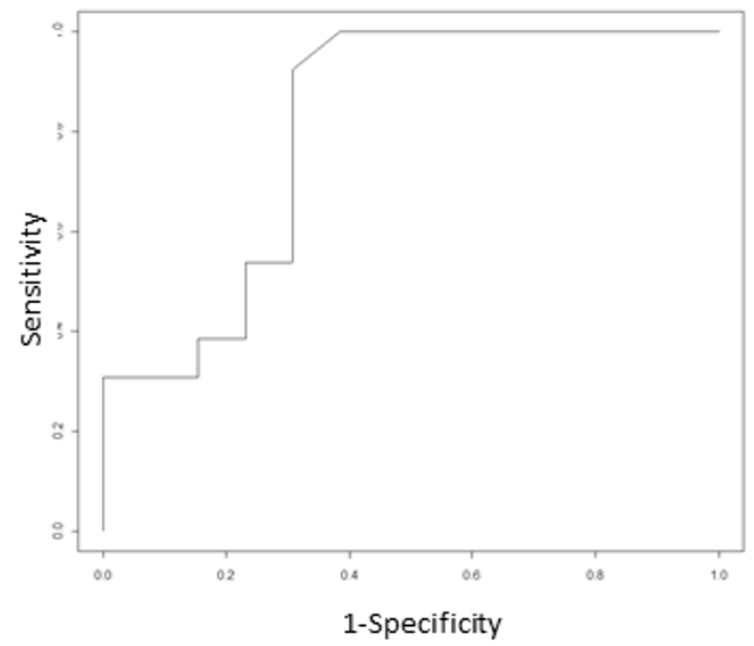

Figure 3 Receiver operating characteristics (ROC) curves for the IRGs as predictor for nonresponse upon RTX treatment in the validation cohort $(\boldsymbol{n}=\mathbf{2 6})$. A. AUC $(0.82)$ for the eight IRG set based on $\triangle D A S 28$ response criteria, B. AUC $(0.87)$ for the three IRG set based on $\triangle \mathrm{DAS} 28$ response criteria, C. AUC (0.78) for the eight IRG set based on EULAR response criteria (responders and intermediate responders vs. nonresponders) and $\mathbf{D}$. AUC (0.83) for the three IRG set based on EULAR response criteria (responders and intermediate responders versus nonresponders). On the $y$-axis sensitivity and on the $x$-axis 1-specificity is indicated. AUC, area under the curve; DAS28, 28 joints disease activity score; EULAR, European League against Rheumatism; IRGs, interferon response genes; RTX, rituximab.

Here, we further extend this initial observation. Firstly, we demonstrated that out of all the genes in the human genome only the IRGs are associated with clinical response to RTX. We identified a set of eight IRGs whose expression status could be translated into a score that showed a significant correlation with response outcome. Secondly, we demonstrated clinical utility by showing that the eight gene-based IFN score predicts the response to RTX using ROC-curve analysis with an AUC of 0.82 in an independent validation cohort. Lastly, advanced data analysis identified a subset of three gene markers that more accurately and robustly predicts the response to RTX therapy (AUC 0.87). For the future, larger and prospective multicenter studies are needed to further validate these findings.

The ROC AUC is an important measure of test accuracy. For the eight gene set assay an AUC of 0.82 was reached, which means that this test correctly classifies $82 \%$ of two patients of randomly drawn pairs, which is considered 'good' [31]. Based on these data a cut-off could be chosen to predict non-response to RTX with a specificity of $100 \%$ and a sensitivity of $44 \%$. Advanced classifier analysis yielded an AUC of 0.87 for a three 
gene test, which is considered close to excellent (AUC > 0.90) at separating responders from non-responders. The association of IRG and RTX outcome appeared independent of other proclaimed biomarkers for RTX treatment. Comparable findings were generated based on the EULAR response outcome measure.

In our study clinical nonresponse at six months was defined by two composite scoring indices: 1) as $\triangle \mathrm{DAS} 28$ $<1.2$ and 2) as EULAR non-response (additionally requires DAS28 to be $>5.1$ ). The use of a composite scoring index could negatively impact the overall response score since individual components (such as joint swelling and tenderness) could be the consequence of irreversible damage in patients who have failed TNFblocking therapy, who are the ones that are treated with RTX. Moreover, a limitation of our study is that we analyzed the predictive value of IRG for RTX response only at six months after initiation of RTX. Although this time point is in line with the time point of the primary outcome of previous randomized controlled trials in RTX-treated RA patients $[8,9]$, we would like to evaluate other time-points as well.

Several investigators have reported on an increased expression of IRGs in peripheral blood cells in a subset of RA patients [17,21,32-35]. Evidence for a role of IFN $\alpha$ and IFN $\beta$ in the induction of IFN response activity in RA was provided by Mavragani and colleagues [33] who demonstrated the concomitant presence of IFN bioactivity, which could be inhibited by neutralizing antibodies against IFN $\alpha$ and IFN $\beta$, in the serum of RA patients. Type I IFNs have an essential function in mediating innate immune responses against viruses and play critical roles in several immunological processes including lymphoid differentiation, homeostasis, tolerance and memory. Although an increased expression of IRGs was associated with the persistence of ACPA after TNF blockade, a direct relationship between IRG expression and autoantibody responses in RA could not be confirmed [36]. Accordingly, we found that the relationship between the IRG activity and response outcome was independent of auto-antibody status.

Results from our study suggest that IFN ${ }^{\text {high }}$ RA patients represent a different pathogenic subset of RA marked by a failure to respond to B-cell depletion therapy. A simple explanation could be that the pathogenesis in IFN ${ }^{\text {high }}$ patients is less dependent on B-cells, compared to IFN ${ }^{\text {low }}$ patients. However, the IRG activity was found to be equally present in seropositive and seronegative RA patients, arguing against an association between IFN-response activity and pathogenic B cells [36]. Alternatively, a high baseline IFN-activity may be associated with the presence of a subset of pathogenic B cells insensitive to the effects of RTX. These could be present at baseline and could survive in synovial or bone marrow tissues due to, for example, incomplete Bcell depletion effectors or concomitant expression of Bcell survival factors, such as BAFF/BLyS [37]. IFNs may also affect B-cell differentiation, such as in situ differentiation in CD20- plasma blasts [38].

Pharmacological studies revealed that the regulation of IRG-activity during treatment is associated with the IFN-response activity prior to the start of treatment [21]. The IRG-activity in non-responders, who have an increased IRG-activity before the start of treatment, remains stable during treatment, whereas good responders, who have a low or absent IFN-response activity at baseline, develop IFN-response activity till a level comparable to that of non-responders during three months of treatment. Thus, baseline IRG-activity appears to be crucial for the pharmacological induction of type I IFNresponse activity, which might be a critical event in the ameliorative effect of B-cell depletion therapy in RA. The increased IFN-activity might explain the increased BAFF/BLyS levels and persistence of pathogenic B cells and could explain the change in macrophage function reflected by an increased BAFF/BLyS, IL10 and CD86 mRNA expression [39].

\section{Conclusions}

In conclusion, we applied genome-wide gene expression technology to identify a set of markers, which accurately and robustly predict the response to RTX treatment in an independent validation group of RA patients. Therefore, these findings are likely to become a substantial aid to the physician, taking the paradigm of personalized medicine one step further.

\section{List of abbreviations}

ACPA: anti-cyclic citrullinated proteins antibodies; AUC: area under curve; Cl: confidence interval; CRP: C reactive protein; DAS28: 28 joints disease activity score; DMARD: disease modifying anti-rheumatic drug; ESR: erythrocyte sedimentation rate; EULAR: European League against Rheumatism; HCQ: hydroxychloroquine; IFN: interferon; IgM: immunoglobuline M; IQR: interquartile range; IRG: interferon response genes; MTX: methotrexate; NSAID: non steroidal anti-inflammatory drug; OR: odds ratio; RA: rheumatoid arthritis; RF: rheumatoid factor; ROC: receiver operating curve; RTX: rituximab; SD: standard deviation; SSZ: sulphasalazyne; TNF: Tumor necrosis factor.

\section{Acknowledgements}

This study was partly supported by grants from the European Community's FP6 funding (AUTOCURE) and the Centre for Medical Systems Biology (CMSB, a center of excellence from the Netherlands Genomics Initiative). This publication reflects only the authors' view. The European Community is not liable for any use that may be made of the information herein.

\section{Author details}

${ }^{1}$ Department of Rheumatology, VU University medical center, de Boelelaan 1117, Amsterdam, 1081HV, the Netherlands. '2Department of Pathology, VU University medical center, de Boelelaan 1118, Amsterdam, 1081 HV, Amsterdam, the Netherlands. ${ }^{3}$ Department of Rheumatology, Jan van Breemen Research Institute|Reade, Jan van Breemenstraat 2, Amsterdam, 1056AB, The Netherlands. " Department of Epidemiology and Biostatistics, VU University medical center, de Boelelaan 1117, Amsterdam, 1081HV, the Netherlands. 


\section{Authors' contributions}

HR was responsible for the acquisition of data, analysis and interpretation of data and drafting of the manuscript. SV performed the RNA experiments, analyzed and interpreted the data and drafted the manuscript. $M N, W L, M B$, $\mathrm{MvdW}, \mathrm{SdR}$, and BD assisted with analysis and interpretation of data and revised the manuscript critically. CV and AV conceived and designed the study, provided technical supervision, analysis and interpretation of the data and revised the manuscript critically. All authors read and approved the final manuscript

\section{Competing interests}

Dr Nurmohamed has received research and speaking fees from Roche Prof. Lems is member of advisory board van Roche, Abbott and MSD. Prof. Dijkmans has received research grants from Wyeth, Abbott and Schering-Plough.

Prof. Verweij is an inventor on a patent application on the predictive value of IFN-type I response activity for the clinical outcome of B-cell depletion therapy. Prof. Boers has received consultation fees of more than $\$ 10,000$ from Roche and less than $\$ 10,000$ from Glaxo Smith Kline, Novartis, AstraZeneca, Abbott, UCB, Schering-Plough and Bristoll-Myers Squibb Mr. Raterman MD, Mr. S. de Ridder MSc, Mrs. Vosslamber MSc, Dr. van der Wiel and Dr. Voskuyl have no competing interests to declare.

Received: 2 May 2011 Revised: 23 February 2012

Accepted: 27 April 2012 Published: 27 April 2012

\section{References}

1. Nielen MM, van Schaardenburg D, Reesink HW, van de Stadt RJ, van der Horst-Bruinsma IE, de Koning MH, Habibuw MR, Vandenbroucke JP, Dijkmans BA: Specific autoantibodies precede the symptoms of rheumatoid arthritis: a study of serial measurements in blood donors. Arthritis Rheum 2004, 50:380-386.

2. Rantapää-Dahlqvist S, de Jong BA, Berglin E, Hallmans G, Wadell G, Stenlund $H$, Sundin $U$, van Venrooij WJ: Antibodies against cyclic citrullinated peptide and IgA rheumatoid factor predict the development of rheumatoid arthritis. Arthritis Rheum 2003, 48:2741-2749.

3. Roosnek E, Lanzavecchia A: Efficient and selective presentation of antigen-antibody complexes by rheumatoid factor B cells. J Exp Med 1991, 173:487-489

4. Kuhn KA, Kulik L, Tomooka B, Braschler KJ, Arend WP, Robinson WH, Holers VM: Antibodies against citrullinated proteins enhance tissue injury in experimental autoimmune arthritis. J Clin Invest 2006, 116:961-973.

5. Lee DM, Friend DS, Gurish MF, Benoist C, Mathis D, Brenner MB: Mast cells: a cellular link between autoantibodies and inflammatory arthritis. Science 2002, 297:1689-1692.

6. Marston B, Palanichamy A, Anolik JH: B cells in the pathogenesis and treatment of rheumatoid arthritis. Curr Opin Rheumatol 2010, 22:307-315.

7. Edwards JC, Leandro MJ, Cambridge G: B lymphocyte depletion therapy with rituximab in rheumatoid arthritis. Rheum Dis Clin North Am 2004, 30:393-403, viii.

8. Cohen SB, Emery P, Greenwald MW, Dougados M, Furie RA, Genovese MC, Keystone EC, Loveless JE, Burmester GR, Cravets MW, Hessey EW, Shaw T, Totoritis MC: Rituximab for rheumatoid arthritis refractory to anti-tumor necrosis factor therapy: results of a multicenter, randomized, doubleblind, placebo-controlled, phase III trial evaluating primary efficacy and safety at twenty-four weeks. Arthritis Rheum 2006, 54:2793-2806.

9. Emery P, Fleischmann R, Filipowicz-Sosnowska A, Schechtman J, Szczepanski L, Kavanaugh A, Racewicz AJ, van Vollenhoven RF, Li NF, Agarwal S, Hessey EW, Shaw TM: The efficacy and safety of rituximab in patients with active rheumatoid arthritis despite methotrexate treatment: results of a phase IIB randomized, double-blind, placebocontrolled, dose-ranging trial. Arthritis Rheum 2006, 54:1390-1400.

10. Quartuccio L, Fabris M, Salvin S, Atzeni F, Saracco M, Benucci M, Cimmino M, Morassi P, Masolini P, Pellerito R, Cutolo M, Puttini PS, De Vita S: Rheumatoid factor positivity rather than anti-CCP positivity, a lower disability and a lower number of anti-TNF agents failed are associated with response to rituximab in rheumatoid arthritis. Rheumatology (Oxford) 2009, 48:1557-1559.

11. Finckh A, Ciurea A, Brulhart L, Moller B, Walker UA, Courvoisier D, Kyburz D, Dudler J, Gabay C: Which subgroup of patients with rheumatoid arthritis benefits from switching to rituximab versus alternative anti-tumour necrosis factor (TNF) agents after previous failure of an anti-TNF agent? Ann Rheum Dis 2010, 69:387-393.

12. Dass S, Rawstron AC, Vital EM, Henshaw K, McGonagle D, Emery P: Highly sensitive $B$ cell analysis predicts response to rituximab therapy in rheumatoid arthritis. Arthritis Rheum 2008, 58:2993-2999.

13. Chatzidionysiou K, Lie E, Nasonov E, Lukina G, Hetland ML, Tarp U, Gabay C, van Riel PL, Nordström DC, Gomez-Reino J, Pavelka K, Tomsic M, Kvien TK, van Vollenhoven RF: Highest clinical effectiveness of rituximab in autoantibody-positive patients with rheumatoid arthritis and in those for whom no more than one previous TNF antagonist has failed: pooled data from 10 European registries. Ann Rheum Dis 2011, 70:1575-1580.

14. Fabris M, Quartuccio L, Lombardi S, Saracco M, Atzeni F, Carletto A, Cimmino M, Fabro C, Pontarini E, Pellerito R, Bambara LM, Sarzi-Puttini P, Cutolo M, Manfredi M, Benucci M, Morassi P, Fischetti F, Padovan M, Govoni M, Curcio F, Tonutti E, De Vita S: The CC homozygosis of the $-174 \mathrm{G}>$ C IL-6 polymorphism predicts a lower efficacy of rituximab therapy in rheumatoid arthritis. Autoimmun Rev 2012, 11:315-320.

15. Magnusson M, Brisslert M, Zendjanchi K, Lindh M, Bokarewa Ml: EpsteinBarr virus in bone marrow of rheumatoid arthritis patients predicts response to rituximab treatment. Rheumatology (Oxford) 2010, 49:1911-1919.

16. Julia A, Barcelo M, Erra A, Palacio C, Marsal S: Identification of candidate genes for rituximab response in rheumatoid arthritis patients by microarray expression profiling in blood cells. Pharmacogenomics 2009, 10:1697-1708.

17. Thurlings RM, Boumans M, Tekstra J, van Roon JA, Vos K, van Westing DM, van Baarsen LG, Bos C, Kirou KA, Gerlag DM, Crow MK, Bijlsma JW Verweij CL, Tak PP: The relationship between the type I interferon signature and the response to rituximab in rheumatoid arthritis. Arthritis Rheum 2010, 62:3607-3614.

18. Arnett FC, Edworthy SM, Bloch DA, McShane DJ, Fries JF, Cooper NS, Healey LA, Kaplan SR, Liang MH, Luthra HS, et al: The American Rheumatism Association 1987 revised criteria for the classification of rheumatoid arthritis. Arthritis Rheum 1988, 31:315-324.

19. Prevoo ML, van 't Hof MA, Kuper $H H$, van Leeuwen $M A$, van de Putte $L B$, van Riel PL: Modified disease activity scores that include twenty-eightjoint counts. Development and validation in a prospective longitudinal study of patients with rheumatoid arthritis. Arthritis Rheum 1995, 38:44-48.

20. van Gestel AM, Prevoo MLL, van't Hof MA, van Rijswijk MH, van de Putte LBA, van Riel PLCM: Development and validation of the European League against Rheumatism response criteria for rheumatoid arthritis: comparison with the preliminary American College of Rheumatology and the World Health Organization/International League against Rheumatism criteria. Arthritis Rheum 1996, 39:34-40.

21. Vosslamber $\mathrm{S}$, Raterman HG, Pouw Kraan T, Schreurs M, Blomberg von M, Nurmohamed MT, Lems WF, Dijkmans BA, Voskuyl A, Verweij C: Pharmacological induction of IFN type I activity following therapy with rituximab determines clinical response in rheumatoid arthritis. Ann Rheum Dis 2011, 70:1153-1159.

22. Bolstad BM, Irizarry RA, Astrand M, Speed TP: A comparison of normalization methods for high density oligonucleotide array data based on variance and bias. Bioinformatics 2003, 19:185-193.

23. Rainer J, Sanchez-Cabo F, Stocker G, Sturn A, Trajanoski Z: CARMAweb: comprehensive R- and bioconductor-based web service for microarray data analysis. Nucleic Acids Res 2006, 34:W498-W503.

24. Eisen MB, Spellman PT, Brown PO, Botstein D: Cluster analysis and display of genome-wide expression patterns. Proc Natl Acad Sci USA 1998, 95:14863-14868.

25. Tusher VG, Tibshirani R, Chu G: Significance analysis of microarrays applied to the ionizing radiation response. Proc Natl Acad Sci USA 2001, 98:5116-5121.

26. Eisen Software. [http://rana.lbl.gov/EisenSoftware.htm].

27. Ingenuity. [http://www.ingenuity.com/products/pathways_analysis.html].

28. Team RDC: R: A language and Environment for Statistical Computing. Vienne Austria: R Foundation for Statistical Computing; 2008.

29. Vince Carey and Henning Redestig for $\mathrm{C}++$ language enhancements: $R O C$ : utilities for ROC, with uarray focus, $\mathrm{R}$ package version 1.26.0

30. Edwards JC, Szczepanski L, Szechinski J, Filipowicz-Sosnowska A, Emery P, Close DR, Stevens RM, Shaw T: Efficacy of B-cell-targeted therapy with rituximab in patients with rheumatoid arthritis. N Engl J Med 2004, 350:2572-2581. 
31. Interpreting Diagnostic Tests. [http://gim.unmc.edu/dxtests/Default.htm].

32. van der Pouw Kraan $T C$, Wijbrandts $C A$, van Baarsen $L G$, Voskuyl $A E$,

Rustenburg F, Baggen JM, Ibrahim SM, Fero M, Dijkmans BA, Tak PP,

Verweij $C L$ : Rheumatoid arthritis subtypes identified by genomic profiling

of peripheral blood cells: assignment of a type I interferon signature in a subpopulation of patients. Ann Rheum Dis 2007, 66:1008-1014.

33. Mavragani CP, La DT, Stohl W, Crow MK: Association of the response to tumor necrosis factor antagonists with plasma type I interferon activity and interferon-beta/alpha ratios in rheumatoid arthritis patients: a post hoc analysis of a predominantly Hispanic cohort. Arthritis Rheum 2010, 62:392-401.

34. Higgs BW, Liu Z, White B, Morehouse C, Brohawn P, Kiener PA, Richman L, Fiorentino D, Greenberg SA, Jallal B, Yao Y: Patients with systemic lupus erythematosus, myositis, rheumatoid arthritis and scleroderma share activation of a common type I interferon pathway. Ann Rheum Dis 2011, 70:2029-2036.

35. O'Hanlon TP, Rider LG, Gan L, Fannin R, Paules RS, Umbach DM, Weinberg CR, Shah RR, Mav D, Gourley MF, Miller FW: Gene expression profiles from discordant monozygotic twins suggest that molecular pathways are shared among multiple systemic autoimmune diseases. Arthritis Res Ther 2011, 13:R69.

36. Cantaert $T$, van Baarsen $L G$, Wijbrandts $C A$, Thurlings $R M$, van de Sande MG, Bos C, van der Pouw TK, Verweij CL, Tak PP, Baeten DL: Type I interferons have no major influence on humoral autoimmunity in rheumatoid arthritis. Rheumatology (Oxford) 2010, 49:156-166.

37. Cambridge G, StohI W, Leandro MJ, Migone TS, Hilbert DM, Edwards JC: Circulating levels of B lymphocyte stimulator in patients with rheumatoid arthritis following rituximab treatment: relationships with $B$ cell depletion, circulating antibodies, and clinical relapse. Arthritis Rheum 2006, 54:723-732.

38. Jego G, Pascual V, Palucka AK, Banchereau J: Dendritic cells control B cell growth and differentiation. Curr Dir Autoimmun 2005, 8:124-139.

39. Toubi E, Kessel A, Slobodin G, Boulman N, Pavlotzky E, Zisman D, Rozenbaum M, Rosner I: Changes in macrophage function after rituximab treatment in patients with rheumatoid arthritis. Ann Rheum Dis 2007, 66:818-820.

doi:10.1186/ar3819

Cite this article as: Raterman et al:: The interferon type I signature towards prediction of non-response to rituximab in rheumatoid arthritis patients. Arthritis Research \& Therapy 2012 14:R95.

\section{Submit your next manuscript to BioMed Central and take full advantage of:}

- Convenient online submission

- Thorough peer review

- No space constraints or color figure charges

- Immediate publication on acceptance

- Inclusion in PubMed, CAS, Scopus and Google Scholar

- Research which is freely available for redistribution

Submit your manuscript at www.biomedcentral.com/submit 